\title{
On the Relationship between the Stratospheric Quasi-biennial Oscillation and the Tropospheric Southern Oscillation
}

\author{
JIN-SONG XU \\ Max-Planck-Institut für Meteorologie, Hamburg, Germany
}

(Manuscript received 5 June 1990, in final form 6 August 1991)

\begin{abstract}
Two quasi-periodic oscillations in the tropical atmosphere with similar oscillation period-the stratospheric quasi-biennial and the Southern oscillations-and the relationship between these two oscillations are examined using the Principal Oscillation Pattern (POP) analysis technique.

The POP analysis of the equatorial stratospheric dataset provides a compact description of the QBO. The oscillation features identified by the POP analysis, namely, the spatial structure, the characteristic times of the oscillation, and the asymmetry in downward propagation, are almost identical to those found by earlier studies using more conventional analyses. The simultaneous POP analysis of the equatorial zonal surface wind and sea surface temperature indicates a well-defined cyclic behavior of the SO. In contrast to the very regular QBO, the SO appears to be much more noisy with intermittent quiet phases. A spectral analysis of the complex POP coefficient time series and the SO index reveals a negligible correlation between the two processes. A POP analysis of the combined equatorial dataset of stratospheric wind, zonal surface wind, and SST also indicates no relation between the QBO and the SO. Two independent modes are identified, one of them completely describing the QBO and the other representing the entire SO. No linear relationship is found between the two modes either in space or in time. It is concluded that the SO and the QBO are two independent processes in the tropical atmosphere with similar time scales.
\end{abstract}

\section{Introduction}

The quasi-biennial oscillation (QBO), which was independently discovered by Reed et al. (1961) and Veryard and Ebdon (1961), is a remarkably regular oscillation of the zonal wind in the equatorial stratosphere. During half of the period of about 26 months, easterlies propagate from the upper stratosphere to the lower stratosphere, and during the other half of the period they are replaced by westerly winds.

The existence of a biennial time scale in the Southern Oscillation (SO) was suggested by Berlage (1955), and was indicated and demonstrated in many studies using longer time series (e.g., Rasmusson and Carpenter 1982; van Loon and Shea 1985, 1987; Gutzler and Harrison 1987; Meehl 1987; Kawamura 1988; and Ropelewski et al. 1987). Xu and Storch (1990) showed that the quasi-biennial cycle is the dominant mode of the SO and that a prediction scheme based on this cycle is skillful in describing and forecasting the SO. Furthermore, Lau and Sheu (1988) and Barnett (1991) suggested that the SO can be understood as a nonlinear interaction between the quasi-biennial time scale and a multiyear time scale.

Corresponding author address: Dr. Jin-Song Xu, Max-Planck-Institut für Meteorologie, Bundesstrasse 55, D 200 Hamburg 13, Germany.
The question now is whether the stratospheric QBO and tropospheric SO are related to each other. Theoretically, it is shown (an overview is given by Holton 1983 ) that the QBO is driven by the interaction between the mean flow and vertically propagating equatorial waves generated in the troposphere (primarily Kelvin and Rossby gravity waves). It is commonly assumed that convective disturbances in the equatorial zone excite these waves in the upper troposphere. The fact that such sources probably have substantial interannual variability related to the SO suggests a possible link between the QBO and the SO. However, this link has not been theoretically proven.

Recently, several observational studies have been done on the relationship between the QBO and the SO. These studies either analyzed directly tropical tropospheric and stratospheric parameters, or examined indirectly the effect of the two oscillations on the Northern Hemisphere winter circulation in the stratosphere. The results of the first group are contradictory: Yasunari (1989) showed evidence of links between the stratospheric and tropospheric zonal winds and the SST anomalies in the tropics, but Quiroz (1983) and Barnett (1991) rejected the idea of a link between the SO and the QBO. The results of the second group indicate the similarity of the stratospheric anomalies brought about by the QBO and the SO. A warm (cold) event, as well as east (west) phase of the QBO (defined by the 50mb equatorial wind), is associated with an anomalously 
weak (strong) stratospheric polar vortex in winter (Quiroz 1983; van Loon and Labitzke 1987; Holton and Tan 1982). However, this fact is not direct proof of a SO-QBO link.

In order to obtain a clear statement about the SOQBO link, a new analysis technique is used in this paper. First, the QBO and the SO are identified as oscillatory modes in the tropical troposphere and stratosphere by means of the POP analysis. In this way, complex indices that control the evolutions of both phenomena are derived. These are then subjected to complex spectral analysis to check the link between the two oscillations. In contrast to the previous studies where only the relationship between one state of the QBO (usually the stratospheric wind on one level, but not the whole signal with its downward propagation feature) and some other parameters was considered, the application of the POP analysis leads to an elegant and compact description of the evolution of both phenomena. The complex spectral analysis of the complex indices, on the other hand, offers exact information about the spectral relationship between two oscillations regardless of the phase difference between them. Therefore, this analysis scheme offers a straightforward and clear interpretation of the SO-QBO relationship.

In some papers, authors refer to the quasi-biennial component of the SO as the "QBO." To avoid any confusion in the present paper, we distinguish between the "Southern Oscillation," which takes place in the tropical troposphere, and the "QBO," describing the quasi-periodic process in the equatorial stratosphere.

The paper is organized as follows. In sections 2 and 3 , analysis techniques and the data are briefly described. In section 4, a complex "POP index" of the QBO is derived from a stratospheric dataset, and the correlation between this index and the traditional Southern Oscillation Index (SOI) is presented. The results of the POP analysis applied to the SO-related tropospheric data are given in section 5 . Finally, in section 6 , the combined dataset of the stratospheric and the SO-related tropospheric data is analyzed with the POP technique. The paper is concluded with a brief discussion in section 7 .

\section{The analysis technique}

\section{a. The POP analysis}

The purpose of the POP analysis (Hasselmann 1988) is to filter out the space-time characteristics of a complex system whose dynamics are unknown or too involved to be easily described. For details, see also Storch et al. $(1988,1990)$. The generalization of the technique for predictive purposes has been described by $\mathrm{Xu}$ and Storch (1990) and Storch and Xu (1990).

The input vector time series $\mathbf{x}(t)$ is assumed to be generated by a first-order multivariate autoregressive process:

$$
\mathbf{x}(t+1)=\mathbf{B x}(t)+\text { noise. }
$$

The matrix $B$ is estimated by $B=C_{1} C_{0}^{-1}$ with $C_{1}$ being the lag- 1 covariance matrix and $C_{0}$ the lag- 0 covariance matrix. The eigenvectors of the system matrix $B$ are called POPs. Generally $\mathbf{B}$ is not symmetric so that the eigenvectors $\mathbf{P}$ and their eigenvalues $\lambda$ are either real or appear in conjugate complex pairs.

In most cases the eigenvectors are (spatially) linearly independent so that any state $\mathbf{x}(t)$ may uniquely be described in terms of the eigenvectors $\mathbf{P}$ :

$$
\mathbf{x}(t)=\sum_{\substack{\text { all } \\ \text { real } \\ \text { POPs }}} z_{j} \mathbf{P}_{j}+\sum_{\substack{\text { all } \\ \text { complex } \\ \text { POPs }}}\left(\frac{1}{2} z_{j} \mathbf{P}_{j}+\frac{1}{2} z_{j}^{*} \mathbf{P}_{j}^{*}\right)
$$

where $z_{j}$ is the coefficient of POP $\mathbf{P}_{j}$, and $z_{j}^{*}$ and $\mathbf{P}_{j}^{*}$ are complex conjugate of $z_{j}$ and $\mathbf{P}_{j}$. For a real POP, the evolution of the coefficients is given by

$$
z\left(t_{0}+t\right)=\lambda^{t} z\left(t_{0}\right)+\text { noise. }
$$

For a complex POP, $\mathbf{P}=\mathbf{P}^{2}+i \mathbf{P}^{1}$, the evolution of the coefficients $z(t)=z^{2}(t)+i z^{1}(t)$ describes a damped clockwise rotation in the complex plane, with superimposed noise:

$$
\begin{aligned}
& z^{2}\left(t+t_{0}\right)+i z^{1}\left(t+t_{0}\right) \\
& \quad=|\lambda|^{t} e^{-(i 2 \pi / T)^{t}}\left[z^{2}\left(t_{0}\right)+i z^{1}\left(t_{0}\right)\right]+\text { noise. }
\end{aligned}
$$

Equation ( $3 \mathrm{~b}$ ) describes the tendency of the system under consideration to generate sequences ... $\rightarrow$ $\mathbf{P}^{1} \rightarrow \mathbf{P}^{2} \rightarrow-\mathbf{P}^{1} \rightarrow-\mathbf{P}^{2} \rightarrow \ldots$ within a certain period $T$ and with an $e$-folding time controlled by $|\lambda|$. Both the $e$-folding time and the oscillation period are functions of the eigenvalue $\lambda$.

According to the assumption made in (1), POPs can also be considered as the normal modes of the original system $\mathbf{x}(t)$. The spatial covariance feature of each mode is described by the POPs, whereas the spectral feature associated with these patterns is described by the corresponding eigenvalues and the coefficients. It will be shown in this paper that the QBO and the SO are dominant modes of the tropical stratosphere and troposphere, respectively, and that there is no one mode for the whole tropical atmosphere.

\section{b. Spectral analysis for complex time series}

In the present paper complex time series are considered, and the degree of spectral correlation of two complex time series has to be assessed. An adequate tool to do so is the complex generalization of the conventional real spectral analysis. This generalization, as suggested by, for example, Gonella (1972), is based on the notion of the decomposition of a complex time series into polarized rotary components.

If $z(\omega)$ is the Fourier component of the complex 
time series $z(t)$, then the complex autospectrum of $z(t)$ is defined as

$$
\Gamma_{z}(\omega)=\left\langle z(\omega) \cdot z(\omega)^{*}\right\rangle .
$$

Here the angle brackets indicate expectation and the symbol * complex conjugate. If $z$ is truly complex, $z(-\omega) \neq z^{*}(\omega)$, and therefore $\Gamma_{z}(\omega) \neq \Gamma_{z}(-\omega)$. Variance attributed to positive (negative) frequencies $\omega$ refers to an anticlockwise (clockwise) rotation. An intriguing aspect of definition (4) is that it is invariant under rotations of the coordinate system, that is, $\Gamma_{z}(\omega)$ $=\Gamma_{a z}(\omega)$ if $a \cdot a^{*}=1$.

The squared coherency spectrum of two complex time series $z(t)$ and $y(t)$ is defined as

$$
C(\omega)=\frac{\left|\left\langle z(\omega) \cdot y(\omega)^{*}\right\rangle\right|}{\left[\Gamma_{z}(\omega) \Gamma_{y}(\omega)\right]^{1 / 2}} .
$$

Similar to the autospectrum, the squared coherency spectrum is not symmetric $(C(\omega) \neq C(-\omega)$; the polarization is defined as for autospectrum) and invariant to rotations of the coordinate system.
The property of rotation invariance makes the complex spectral analysis a suitable technique for the present study. Considering two complex coefficients $z_{i}$ and $z_{j}$ of two complex POPs and assuming $z_{i}=c z_{j}$ with $c$ $=\alpha e^{i \phi}$, a conventional correlation technique cannot detect any correlation relationship between real parts of $z_{i}$ and $z_{j}$ and between imaginary parts of $z_{i}$ and $z_{j}$ if $\phi=2 / \pi$. In contrast, the correlation relationship between two oscillations described by $z_{i}$ and $z_{j}$ can be clearly demonstrated by the complex spectral analysis regardless of $\phi$. The autospectrum and the squared coherency spectrum may be estimated in a conventional manner using the Bartlett procedure.

\section{Data}

\section{a. Stratospheric data}

The equatorial zonal wind exhibits only small longitudinal variations, and therefore, the zonal mean wind may conveniently be estimated from station data. Using the observations from three stations:

$$
\begin{array}{lll}
\text { Canton Island } & 2^{\circ} 46^{\prime} \mathrm{S}, 171^{\circ} 43^{\prime} \mathrm{W} & \text { Jan 1953-Aug } 1967 \\
\text { Gan, Maldive Islands } & 0^{\circ} 41^{\prime} \mathrm{S}, 73^{\circ} 09^{\prime} \mathrm{E} & \text { Sep 1967-Dec } 1975 \\
\text { Singapore } & 1^{\circ} 22^{\prime} \mathrm{N}, 103^{\circ} 55^{\prime} \mathrm{E} & \text { Jan 1976-Aug } 1989
\end{array}
$$

time series of stratospheric zonal wind were compiled by the Institute for Meteorology of the Free University Berlin (Naujokat 1986). Complete time series for the entire period 1953-1989 are available at six levels: 70, $50,40,30,20$, and $15 \mathrm{mb}$. The long-term mean flow is easterly with a vertical average of about $-3.9 \mathrm{~m} \mathrm{~s}^{-1}$. In the following analysis, deviations from this longterm mean are considered. No time filtering was done for this dataset.

\section{b. SO-related data}

The combined dataset of monthly mean equatorial zonal wind anomalies at the surface and the SST anomalies from $45^{\circ} \mathrm{E}$ to $85^{\circ} \mathrm{W}$ has been found to contain a well-defined SO signal (Storch et al. 1990; Barnett 1983). With respect to the wind, the signal appears to be eastward propagating, and with respect to SST to be almost standing. This dataset, covering the time period 1951-1986, is also used in the present study. To remove high-frequency noise, the data are low-pass filtered (the filter characteristic is the same as described in Xu and von Storch 1990). In the combined dataset, the low-pass-filtered wind and the low-pass-filtered SST are both normalized so that the variances in both parameters are comparable.

To investigate the relationship between the $\mathrm{QBO}$ and the SO, the traditional Southern Oscillation Index (SOI) - pressure anomaly difference between Darwin and Tahiti-is also used. This time series was low-pass time filtered in the same manner as the surface wind and the SST.

\section{The $Q B O$ and the equatorial stratosphere}

The POP analysis of the stratospheric wind anomalies reveals one physically significant POP pair with an oscillation period of about 27 months. It is shown in this section that this POP represents the traditional stratospheric QBO. The coefficient time series, $z^{1}(t)$ and $z^{2}(t)$, and the patterns, $\mathbf{P}^{1}$ and $\mathbf{P}^{2}$, are shown in Figs. 1a and 2a.

\section{a. Average features of the $Q B O$}

Apart from minor high-frequency variations, the coefficient time series are characterized by coherent variations on a quasi-biennial time scale. The two time series are almost $90^{\circ}$ out of phase throughout the entire data interval, indicating a permanent oscillation in the stratosphere.

According to the POP concept, the pattern $\mathbf{P}^{\mathrm{T}}$ is replaced by the pattern $\mathbf{P}^{2}$ within a quarter of the oscillation period. Since the maximum wind in the pattern $\mathbf{P}^{1}$ is at $20 \mathrm{mb}$ (Fig. 2a) and in $\mathbf{P}^{2}$ at $40 \mathrm{mb}$, this replacement describes the downward propagation of the maximum wind from $20 \mathrm{mb}$ to $40 \mathrm{mb}$. In $\mathbf{P}^{2}$, there 
a

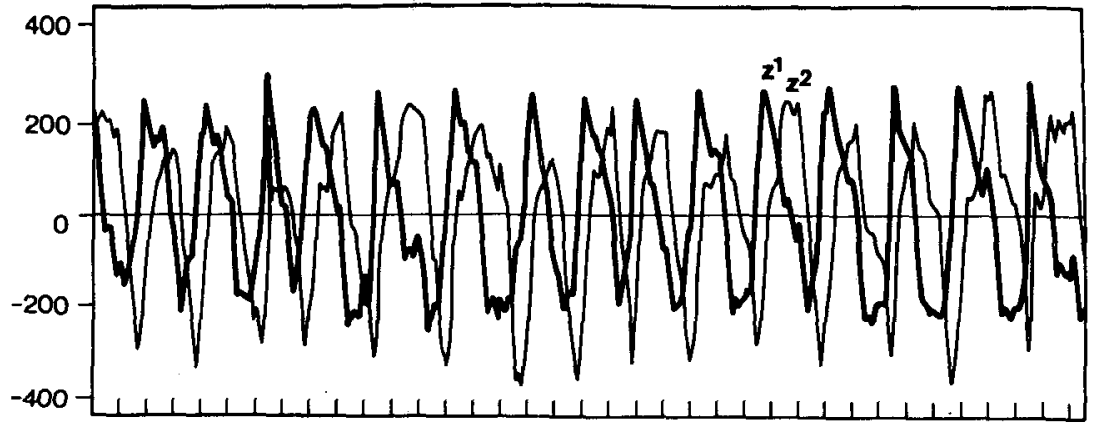

b

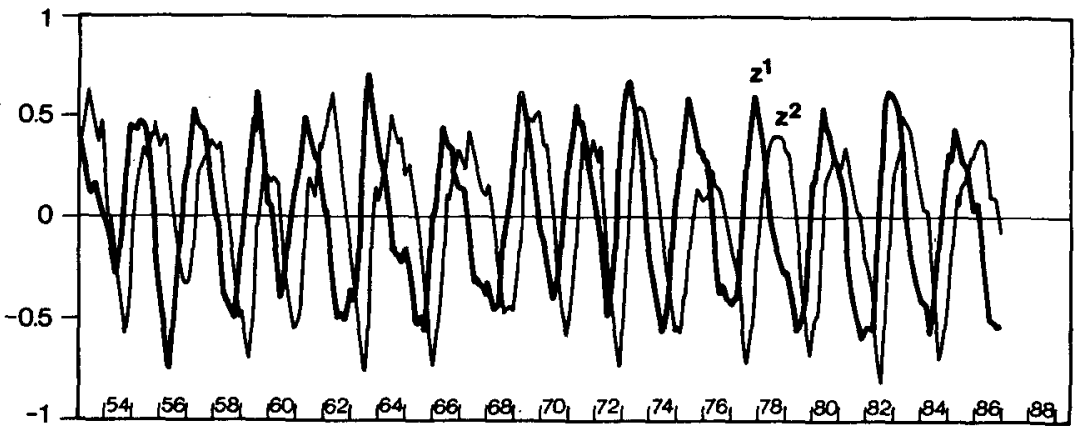

FIG. 1. POP coefficient time series (in relative units) from (a) POP analysis for the unfiltered stratospheric zonal wind anomalies; (b) the first mode of the POP analysis for the combined dataset including stratospheric zonal wind and surface zonal wind and SST anomalies.
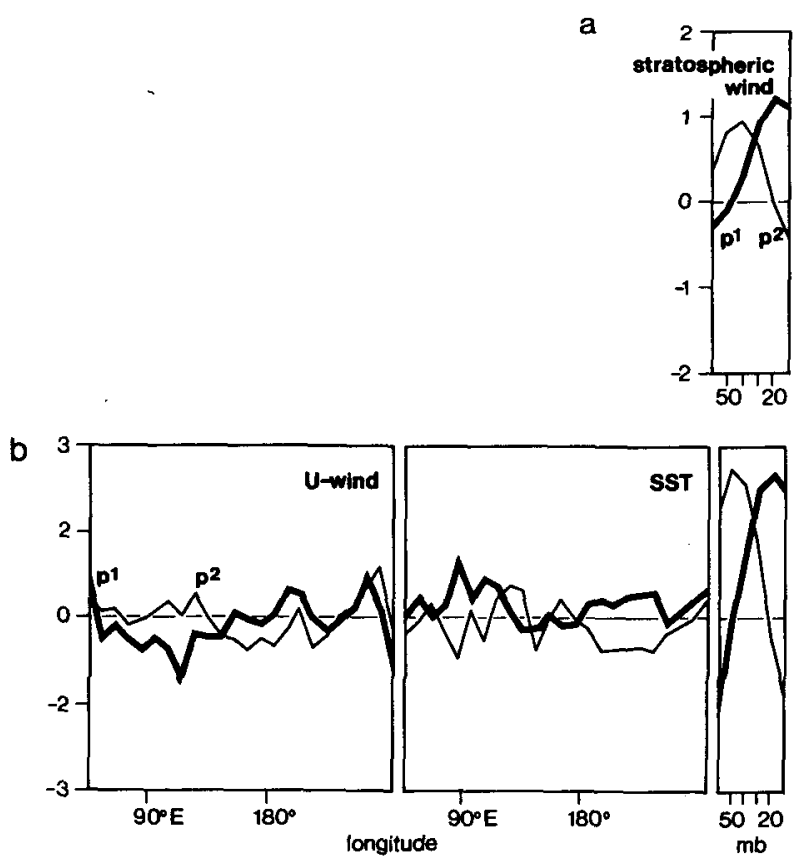

FIG. 2. POP patterns $P^{1}$ and $P^{2}$ (in relative units) from (a) POP analysis for the unfiltered stratospheric zonal wind anomalies; (b) the first mode of the POP analysis for the combined dataset including the stratospheric zonal wind and surface zonal wind and SST anomalies. is also a minor minimum at $20 \mathrm{mb}$. The transition from $\mathbf{P}^{2}$ to $-\mathbf{P}^{1}$ describes the intensification of this minimum, and therefore the rebuilding of the maximum at $20 \mathrm{mb}$ with an opposite wind.

The state of the QBO at a certain time $t$ is given by $z^{1}(t) \mathbf{P}^{1}+z^{2}(t) \mathbf{P}^{2}$; that is, the POP coefficients $z^{1}$ and $z^{2}$ form a complex index $z$ of the QBO. The downward propagation of the westerlies (easterlies) is described by positive (negative) $z^{1}$ and $z^{2}$. The transition from westerly to easterly (easterly to westerly) is described by positive (negative) $z^{1}$ and negative (positive) $z^{2}$.

\section{b. Deviation from the biennial time scale}

Sometimes the POP coefficient time series deviates markedly from the regular quasi-biennial progression. For example, in Fig. 1a the westerly regime in 1959 appears fairly short but those in 1966/67 and 1987/ 88 are relatively long; similarly, the easterlies in 1972 lasted shorter than on the average, but those in 1964/ 65 and 1967/68 prevailed longer than usual. These deviations from the regular oscillation coincide with Naujokat's (1986) findings.

\section{c. Asymmetries of the $Q B O$}

The scatter diagram of the normalized complex POP index $\mathrm{z}$ (Fig. 3 ) reveals a characteristic asymmetry be- 


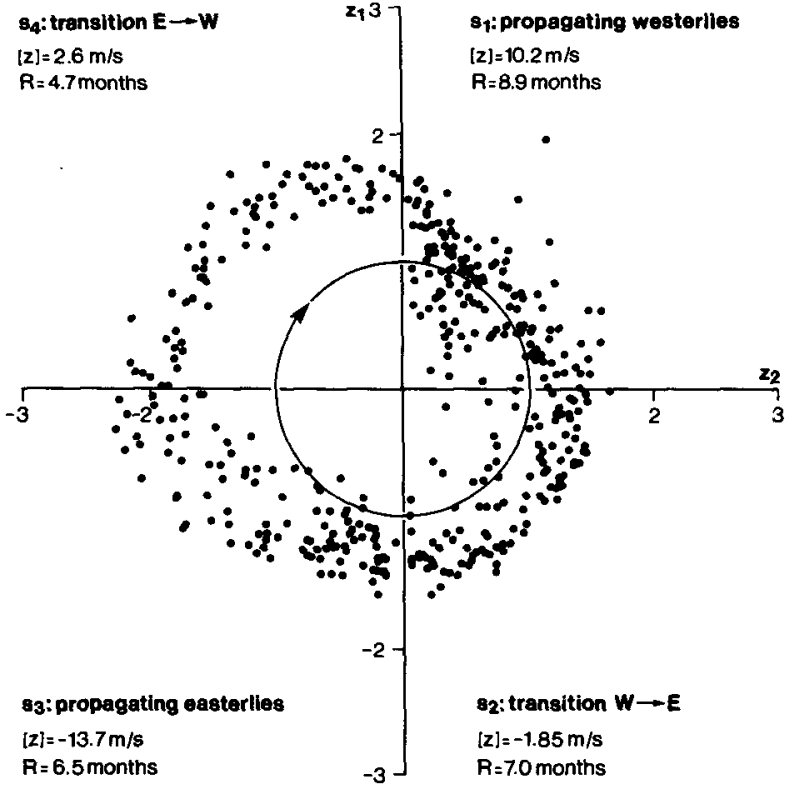

FIG. 3. Distribution of the complex POP index $z$ of the $Q B O$ in the complex $z^{1}-z^{2}$ plane. The unit circle, the vertical average $[z]$ of $z^{1} \mathbf{P}^{1}+z^{2} \mathbf{P}^{2}$ (in $\mathrm{m} \mathrm{s}^{-1}$ ), and the mean residence time $R$ (in months) during which the index stays in one of the four quarters $S_{i}$ are given to help the reader to assess the asymmetry of the distribution.

tween the west and east phases of the QBO. The distribution is not circular but is elliptical.

To quantify this asymmetry, four $90^{\circ}$ sectors in the complex $z^{1} / z^{2}$ plane are defined, and two characteristic numbers are derived for each of the sectors. The sectors are

$S_{1}: z^{1} \geqslant 0, z^{2} \geqslant 0$ downward propagation of westerlies;

$S_{2}: z^{1} \leqslant 0, z^{2} \geqslant 0$ transition from a westerly regime to an easterly regime;

$S_{3}: z^{1} \leqslant 0, z^{2} \leqslant 0$ downward propagation of easterlies;

$S_{4}: z^{1} \geqslant 0, z^{2} \leqslant 0 \quad$ transition from an easterly regime to a westerly regime.

The characteristic numbers are

[z] defined as the vertical average (in $\mathrm{m} \mathrm{s}^{-1}$ ) of the signal $z^{1}(t) \mathbf{P}^{1}+z^{2}(t) \mathbf{P}^{2}$

$R$ defined as the mean residence time (in months) of the trajectory in the considered sector. It is approximated by $R=(n / m) \cdot T$ with $n$ $=$ number of points $z(t)$ in the sector, $m$ $=$ number of all points $z(t)$, and $T=$ oscillation period. The propagation speed of the signal within a $90^{\circ}$ sector is inverse to its residence time.

The asymmetries of the QBO are

1) The easterlies in $S_{3}$ are stronger than the westerlies in $S_{1}\left(-[z]_{3} \geqslant[z]_{1}\right)$.
2) The downward propagation of the westerlies from $20 \mathrm{mb}$ to $40 \mathrm{mb}$ in $\mathrm{S}_{1}$ is slower than that of the easterlies in $\mathrm{S}_{3}\left(R_{1} \geqslant R_{3}\right)$.

3) The transition phase $S_{4}$ is generally shorter than the transition phase $\mathrm{S}_{2}\left(R_{4} \leqslant R_{2}\right)$.

4) The second-moment statistics of the QBO are not uniform in the four sectors. The distribution of $\mathbf{z}$ in $S_{1}$ is noisy; that is, the downward propagation of the westerlies exhibits an appreciable interannual variability. In $\mathrm{S}_{4}$, on the other hand, the trajectory varies only little from cycle to cycle-the transition from easterly to westerly wind happens in a remarkably regular manner.

Most of the results presented in this section can also be observed by studying the time-height diagram of the equatorial zonal wind (Naujokat 1986). The asymmetry of the QBO was also mentioned by Maruyama and Tsuneoka (1988). The advantage of the present approach is that the POP technique offers more compact, detailed, and exact statistics of the QBO.

\section{d. Relationship between the bivariate index of the $Q B O$ and the traditional Southern Oscillation index}

The autospectra of the complex POP index $z_{\mathrm{QBO}}$, derived from the QBO data, and of the real-valued SOI, as well as the squared coherency spectrum between the two time series, are shown in Fig. 4.

The symmetric autospectrum of the SOI (dashed line in Fig. 4a) has a broad maximum at a time scale of about 2-7 years, whereas the nonsymmetric autospectrum of $\mathrm{z}$ (solid line in Fig. 4a) has a marked peak at $\omega=1 /(-30$ months $)$. The squared coherency spec-
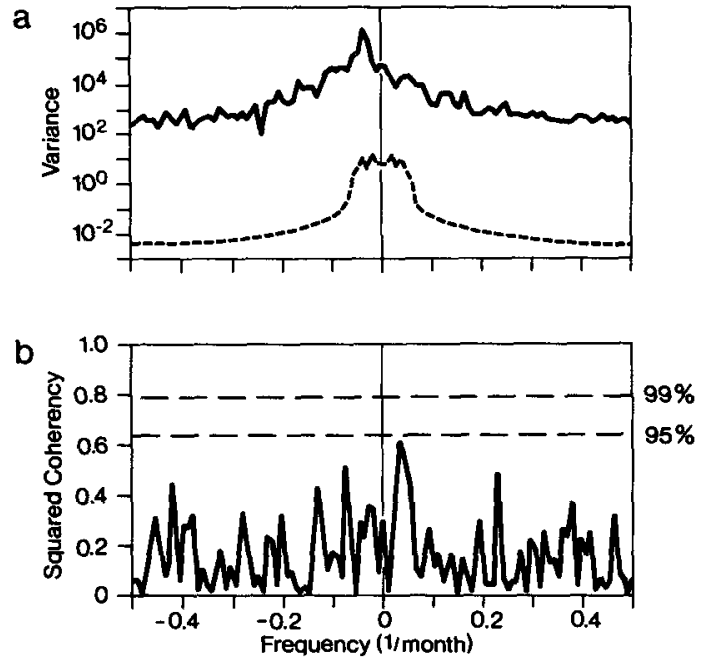

Fig. 4. Spectral analysis of the complex POP coefficient $\mathrm{z}_{\mathrm{QBO}}$, representative of the QBO shown in Fig. 1a, and of the SOI. (a) Autospectra of the POP coefficient (solid) and of the SOI (dashed); (b) squared coherency spectrum of the two time series. 
trum (Fig. 4b) indicates no spectral correlation between the two time series at any time scale.

It is concluded that there is no linear correlation relationship between the $\mathrm{SO}$ and the $\mathrm{QBO}$, even if both processes share a common time scale of about 30 months. The $\mathrm{QBO}$ is a fairly regular phenomenon with only one spectral peak, whereas the SO appears as a phenomenon with a broader spectral band.

\section{The SO and the anomalies of zonal surface wind and SST}

One physically significant POP pair is found in the joint dataset of surface zonal wind and SST anomalies. The oscillation period derived from the POP analysis is about $\mathbf{4 2}$ months. The coefficient time series, $z^{i}(t)$ and $z^{2}(t)$, and the patterns $\mathbf{P}^{1}$ and $\mathbf{P}^{2}$ are shown in Figs. 5a and 6a. Anticipating the results presented in this section, the process described by the POPs is called the Southern Oscillation (SO).

\section{a. Temporal and spatial evolution of SO in the surface zonal winds and SST}

The POP coefficients $z^{1}(t)$ and $z^{2}(t)$ shown in Fig. 5 a describe a regular oscillation in 1963-66, 1970-76, and 1982-83, when either a sequence of warm and cold events or one extreme of the SO occurred. During these periods the two time series are nearly $90^{\circ}$ out of phase. The signal is not very clear in the 1950 s. The POP coefficient time series demonstrate conclusively that the SO may be considered to be an oscillation. However, the SO is less uniform than the QBO, and appears in varied sequences of events instead of the uninterrupted sequence of oscillations that the $\mathrm{QBO}$ does.

In the pattern $\mathbf{P}^{1}$, easterly wind anomalies over the central Pacific and westerly wind anomalies over the Indian Ocean are connected with large negative SST anomalies over the east and central Pacific, positive SST over Indonesia, and negative SST over the Indian Ocean (Fig. 6a). According to the POP concept and in accordance with the characteristic time lag of the POP coefficients, the pattern $\mathbf{P}^{1}$ will be replaced by $\mathbf{P}^{2}$ after a quarter of a period, that is, when the westerly anomalies move into the west Pacific. Simultaneously the SST anomalies in the central and east Pacific are weakened and the SST anomalies in the Indonesia region are shifted somewhat eastward. This state can be regarded as the precursor phase of a warm event. After another quarter of a period, westerly anomalies appear over the central Pacific and positive SST anomalies in the central and eastern tropical Pacific. This is described by $-\mathbf{P}^{1}$, that is, the mature phase of a warm event.

The state of the oscillation can again be presented in the complex $z^{1} / z^{2}$ plane. In Fig. 7 the normalized a

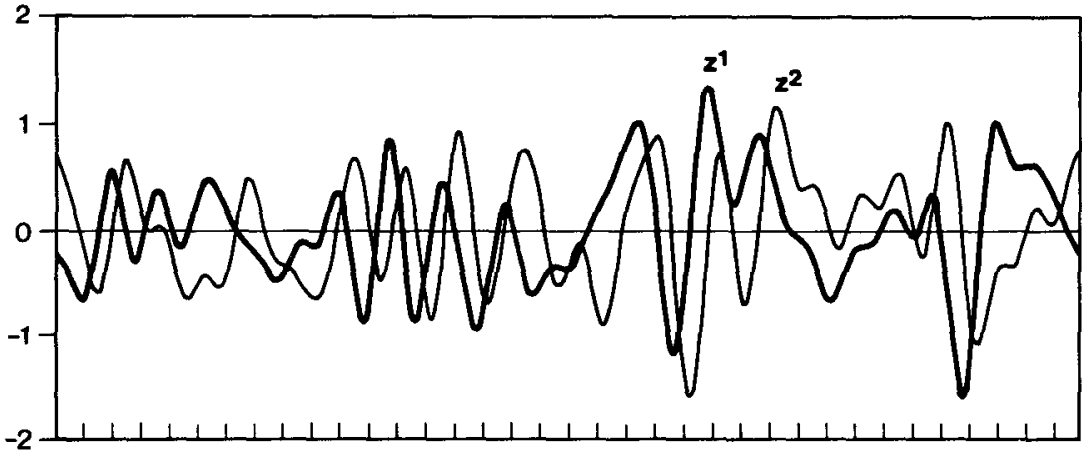

b

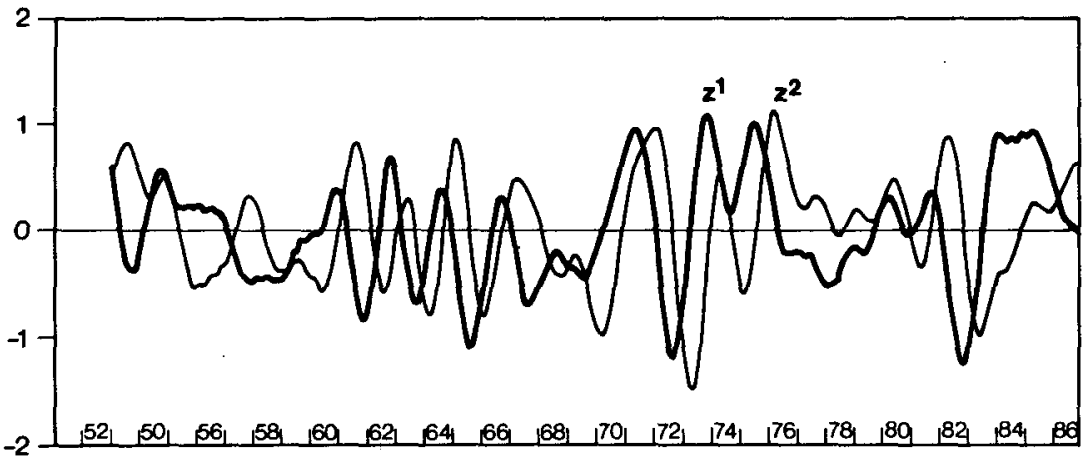

FIG. 5. POP coefficient time series (in relative units) from (a) POP analysis for the filtered surface zonal wind and SST anomalies; (b) the second mode of the POP analysis for the combined dataset including the stratospheric zonal wind and surface zonal wind and SST anomalies. 
a
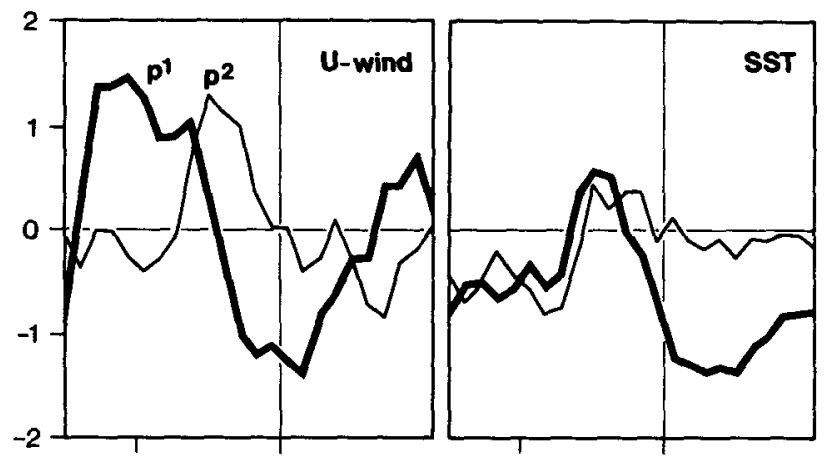

b

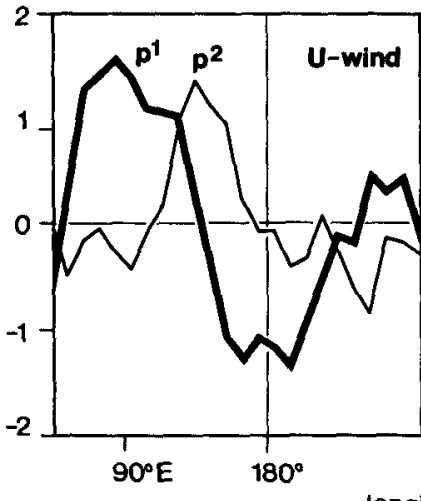

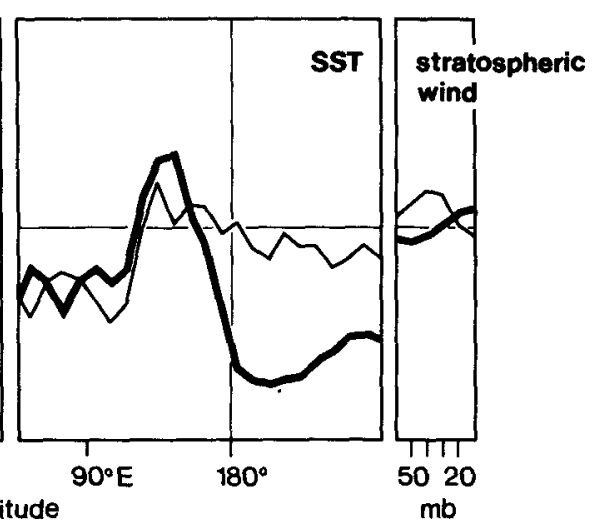

FIG. 6. POP patterns $P^{1}$ and $P^{2}$ (in relative units) from (a) POP analysis for the filtered surface zonal wind and SST anomalies; (b) the second mode of the POP analysis for the combined dataset including the stratospheric zonal wind and surface zonal wind and SST anomalies.

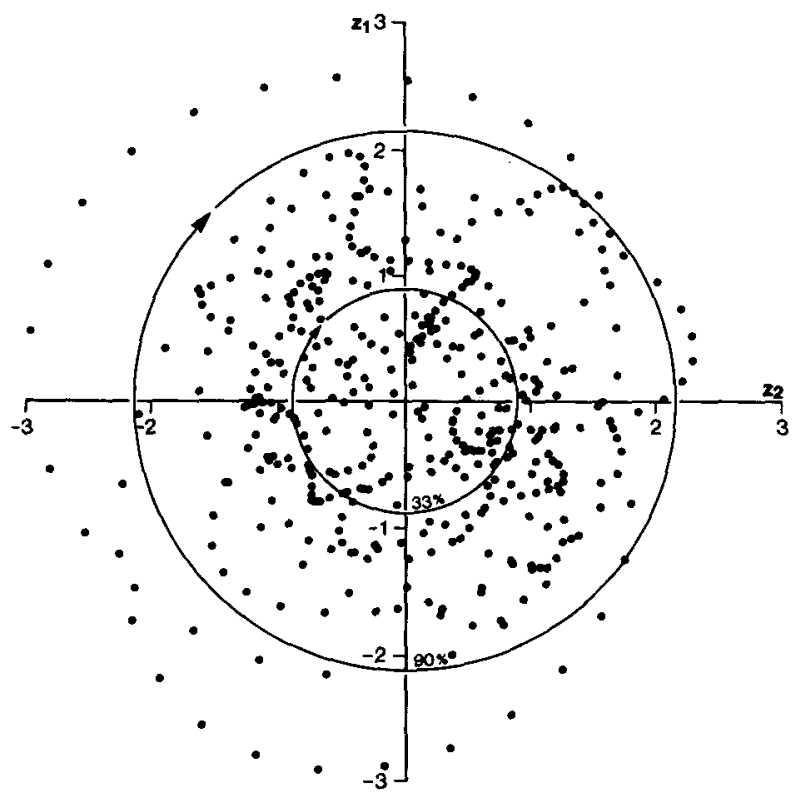

FIG. 7. Distribution of the complex POP index $z$ of the SO in the $z^{i}-z^{2}$ plane. The $30 \%$ and $90 \%$ circle of a binormal distribution are given to assess distribution features.
POP coefficient $\mathbf{z}_{\text {SoI }}$ is plotted. The $\mathbf{z}_{\text {soI }}$ distribution is binormal with maximum density at the origin of the complex plane. It is completely different from the distribution of $\mathrm{z}_{\mathrm{QBO}}$, which exhibits a minimum at the origin and whose maxima form a somewhat distorted circle (Fig. 3). The different distributions indicate different behaviors of the two phenomena. Instead of permanent oscillation as in the case of the QBO, the SO is sometimes in a quiet phase with small amplitude of $z_{\text {sOl }}$, and sometimes reveals oscillatory behavior, shown by the large cyclic path. The oscillation itself is irregular with different durations (for details see $\mathrm{Xu}$ and Storch 1990).

\section{b. Spectral characteristics of the bivariate index of the SO}

The autospectra of the complex POP coefficient $\mathbf{z}_{\text {SOI }}$ derived from surface wind and SST, and that of the real-valued SOI and the squared coherency spectrum between these two time series, are shown in Fig. 8. The autospectra, both of the SOI (dashed line in Fig. 8a) and of the complex POP coefficient (solid line in Fig. 8a), show large variances at a time scale of about 
a
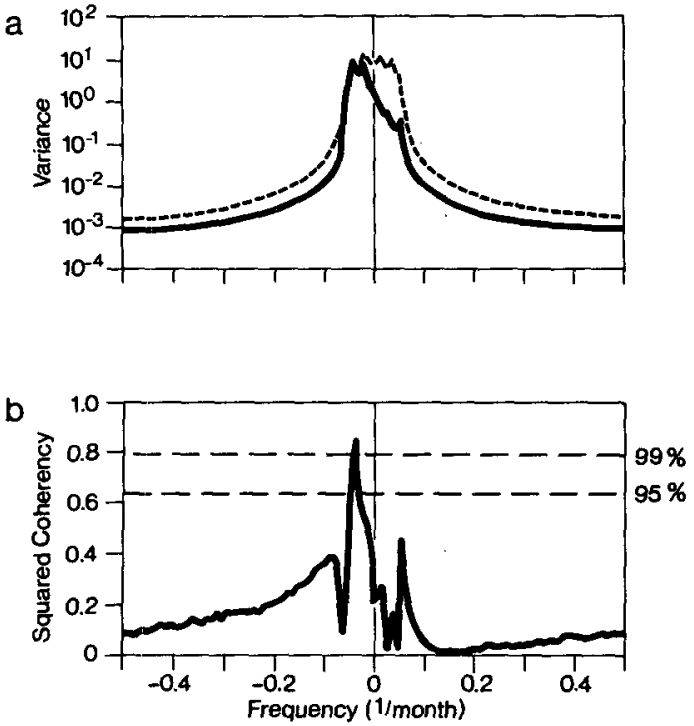

FIG. 8. Spectral analysis of the complex POP coefficient $z_{\text {sol }}$, representative of the SO shown in Fig. 5a, and of the SOI. (a) Autospectra of the POP coefficient (solid) and of the SOI (dashed); (b) squared coherency spectrum of the two time series.

$\omega=1 /(-30$ to -50 months $)$. The squared coherency spectrum (Fig. 8b) confirms the significant correlation relationship at these time scales. Here $z^{1}$ is about $90^{\circ}$ out of phase compared to $z^{2}$ (phase spectrum is not shown). Because of this out-of-phase relationship of the POP coefficient, the complex SO index $\mathbf{z}_{\text {SOI }}$ is capable of monitoring not only the extreme phase of the SO, but also the precursor or transition phase of the SO (cf. Xu and Storch 1990).

\section{Simultaneous analysis of the $\mathrm{QBO}$ and the $\mathrm{SO}$}

In this section the two datasets separately considered in the last two sections-stratospheric wind and SO- related surface data-are simultaneously $P O P$ analyzed. The three components are normalized so that surface wind, SST, and stratospheric wind contribute the same variance to the combined dataset. The analysis is done with data for 1953-1986, which is the overlap interval of the two datasets. Two significant POP pairs are found, one with an oscillation period of 28 months, the other with 45 months.

The POP coefficient of the first mode is shown in Fig. 1b. The time series are almost identical to the QBO POP coefficient time series in Fig. 1a. The stratospheric part of the patterns of mode 1 (Fig. 2b) are very similar to the QBO patterns (Fig. 2a) that describe the downward propagation of wind maximum. The large maximum at lower levels is due to the normalization for the combined dataset. The surface wind and SST components of this POP pair are small and noisy.

For the second mode, POPs and their coefficients are shown in Figs. $6 \mathrm{~b}$ and $5 \mathrm{~b}$. Both the pattern and the time series are almost identical to those found in section 5 for the POP analysis using surface wind and SST only. That part of the patterns that describes the stratospheric wind has very small amolitude.

Figure 9 shows also that the first mode explains a considerable percentage of variance $(40 \%-50 \%)$ only in the stratospheric part of the combined dataset, whereas the second mode explains a considerable percentage of variance $(15 \%-30 \%)$ only in the tropospheric part of the dataset. Undoubtedly, the first and second POP of the combined dataset represent completely and exclusively the $\mathrm{QBO}$ and the $\mathrm{SO}$, respectively. We cannot find a mode that describes the whole tropical atmosphere.

The autospectra and squared coherency spectrum of these complex POP coefficients are shown in Fig. 10. Both autospectra have a maximum at periods around $-\mathbf{3 0}$ months, corresponding to the clockwise oscillation demonstrated in Figs. 3 and 7. These two oscillations are not correlated to each other, as shown

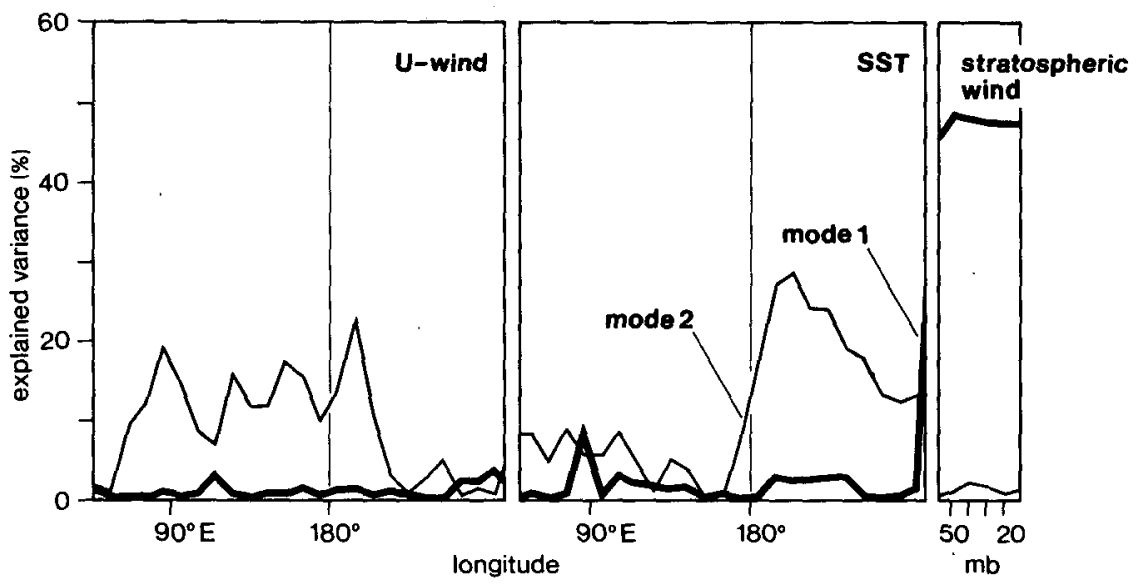

FIG. 9. Percentage of variance of the unfiltered data explained by the first mode (the mode described in Fig. 2b) and by the second mode (the mode described in Fig. 6b). 

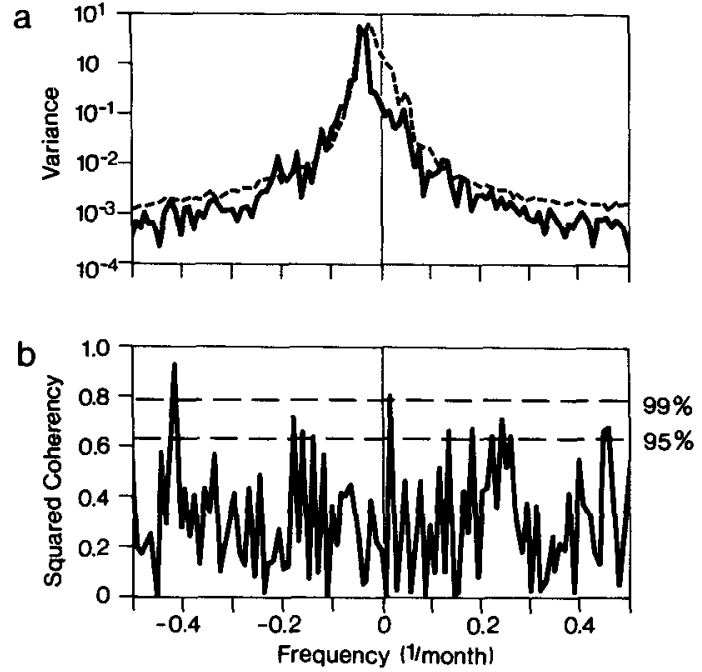

FIG. 10. Spectral analysis of the complex POP coefficients for the first and second modes in the combined dataset shown in Fig. 1b and Fig. 5b. (a) Autospectra of the POP coefficient for the first mode (solid) and for the second mode (dashed); (b) squared coherency spectrum of the two time series.

by the low squared coherency at periods from -20 to -50 months. Obviously there is no direct relationship between the stratospheric QBO and the tropospheric SO.

\section{Discussion and conclusions}

The POP analysis is a powerful technique for capturing spatial and temporal characteristics of a complex system. The examination of stratospheric and tropospheric tropical data with the POP analysis leads to a compact description of the QBO and the SO.

The POP coefficients that monitor the QBO and the SO have significantly different distributions. The SO distribution is binormal with maximum density in the center of gravity. The QBO, however, is not normally distributed; the maxima of density form a distorted circle. The differences between the two POP coefficients indicate that the SO is characterized by sequences of events with irregular breaks, whereas the QBO appears as an uninterrupted sequence of oscillations.

Spatially the eastward propagation of equatorial zonal wind anomalies ( $\mathrm{SO}$ ) associated with warming and cooling in the central and eastern Pacific proceeds independently of the downward propagation of the equatorial stratospheric zonal wind (QBO). The spatial variations associated with the SO (QBO) explain only a negligible amount of variance in the stratosphere (troposphere).

It is concluded that the QBO and the SO are two independent modes of the tropical atmosphere. This statement is a straightforward interpretation of the re- sults from the POP analysis and complex spectral analysis.

Evidence about the poor relationship between the SO and the QBO has also been reported by other researchers on the subject. Barnett (1991) found that there is no significant correlation between the stratospheric wind and the quasi-biennial component of the SO for any lead/lag time. Quiroz (1983) investigated the relation between several stratospheric and tropospheric parameters and found that "coherences significant at the $95 \%$ and $90 \%$ level appear for all pairs of variables which do not involve the $\mathrm{QBO}$. The lowest coherence found was for the QBO and the Southern Oscillation index." He proposed that "since data at only one level, $30 \mathrm{mb}$, were used to represent the QBO, any further investigation should probably take altitude and QBO phase and period variations into account, as well as regional components of the Southern Oscillation." That is exactly what has been done in this paper, and no signal has been found.

The poor relationship between the QBO and the SO represented here contrasts with Yasunari (1989), who claimed to have found a relationship between the SO and QBO. His study relies on QBO bandpass filtering and a relatively short time series of only 15 years. It is therefore suspected that his result may be at least in part an artifact of his analysis technique.

Acknowledgments. I wish to express my gratitude to Barbara Naujokat and Tim Barnett for supplying me with the stratospheric dataset and the equatorial surface zonal winds and SST, and to Klaus Hasselmann, Hans v. Storch, Karin Labitzke, and Harry van Loon for helpful discussion and encouragement. Thanks are also due to Adrian Gordon and Roland Byron-Scott for stimulating discussion and for help with the English text.

\section{REFERENCES}

Barnett, T. P., 1983: Interaction of the monsoon and Pacific trade wind system at interannual time scales. Part I: The equatorial zone. Mon. Wea. Rev., 111, 756-773.

- 1991: The interaction of multiple time scales in the tropical climate system. J. Climate, 4, 269-285.

Berlage, H. P., 1955: The Southern Oscillation-A 2-3 year fundamental oscillation of world-wide significance, I.U.G.G. 10th General Assembly, Rome 1954. Scientific Proc. of the International Association of Meteorology, London, 336-345.

Gonella, J., 1972: A rotary-component method for analyzing meteorological and oceanographic vector time series. Deep-Sea Res., 19, 855-846.

Gutzler, D. S., and D. E. Harrison, 1987: The structure and evolution of seasonal wind anomalies over the near-equatorial eastern Indian and western Pacific Oceans. Mon. Wea. Rev., 115, 169192.

Hasselmann, K., 1988: PIPs and POPs: the reduction of complex dynamical systems using principal interaction and oscillation patterns. J. Geophys. Res., 93, $11015-11021$.

Holton, J. R., 1983: The stratosphere and its links to the troposphere. Large-Scale Dynamical Processes in the Atmosphere, B. Hoskins and R. Pearce, Eds., Academic Press, 277-303. 
and H-C. Tan, 1982: The quasi-biennial oscillation in the Northern Hemisphere lower stratosphere. J. Meteor. Soc. Japan, 60, 140-148.

Kawamura, R., 1988: Quasi-biennial Oscillation modes appearing in the tropical sea water temperature and $700 \mathrm{mb}$ zonal wind. J. Meteor. Soc. Japan, 66, 955-965.

Lau, K.-M., and P. J. Sheu, 1988: Annual cycle, Quasi-biennial Oscillation, and the Southern Oscillation in global precipitation. J. Geophys. Res. 93, 10 975-10988.

Maruyama, T., and Y. Tsuneoka, 1988: Anomalously short duration of the easterly wind phase of the QBO at $50 \mathrm{hPa}$ in 1987 and its relationship to an EI Niño event. J. Meteor. Soc. Japan, 66, 629-633.

Meehl, G., 1987: The annual cycle and the interannual variability in the tropical Pacific and Indian Ocean regions. Mon. Wea. Rev., 115, 27-50.

Naujokat, B., 1986: An update of the observed Quasi-biennial Oscillation of the stratospheric winds over the tropics. J. Atmos. Sci., 43, 1873-1877.

Quiroz, R. S., 1983: Relationships among the stratospheric and tropospheric zonal flows and the Southern Oscillation. Mon. Wea. Rev., 111, 143-154.

Rasmusson, E. M., and T. H. Carpenter, 1982: Variations in tropical sea surface temperature and surface wind fields associated with the Southern Oscillation/El Niño. Mon. Wea. Rev., 110, 354384.

Reed, R. J., W. J. Campbell, L. A. Rasmussen, and D. G. Rogers, 1961: Evidence of downward propagating annual wind reversal in the equatorial stratosphere. J. Geophys. Res., 66, 813-818.

Ropelewski, C. F., M. H. Halpert, and E. M. Rasmusson, 1987: Tropospheric biennial variability and its relationship to the Southern
Oscillation. Twelfth Annual Climate Diagnostics Workshop, Salt Lake City, Utah.

Storch, H. v., and J. Xu, 1990: Principal oscillation pattern analysis of the tropical 30- to 60-day oscillation. Part I: Definition of an index and its prediction. Climate Dyn., 4, 175-190.

$\longrightarrow$ T. Bruns, I. Fischer-Bruns, and K. H. Hasselmann, 1988: Principal oscillation pattern analysis of the 30 to 60 day oscillation in a GCM. J. Geophys. Res., 93, 11 022-11 036.

$\longrightarrow$ U. Weese, and J. Xu, 1990: Simultaneous analysis of spacetime variability: Principal oscillation patterns and principal interaction patterns with applications to the Southern Oscillation. Z. Meteor., 40, 99-103.

van Loon, H., and D. J. Shea, 1985: The Southern Oscillation. Part IV: The precursors south of $15^{\circ} \mathrm{S}$ to the extremes of the oscillations. Mon. Wea. Rev., 113, 2063-2074.

$\longrightarrow$, and - 1987: The Southern Oscillation. Part VI: Anomalies of sea level pressure on the Southern Hemisphere and of Pacific sea surface temperature during the development of a warm event. Mon. Wea. Rev., 115, 370-379.

—, and K. Labitzke, 1987: The Southern Oscillation. Part V: The anomalies in the lower stratosphere of the Northern Hemisphere in winter and a comparison with the Quasi-biennial Oscillation. Mon. Wea. Rev., 115, 357-369.

Veryard, R. G., and R. A. Ebdon, 1961: Fluctuations in tropical stratospheric winds. Meteor. Mag., 90, 125-143.

$\mathrm{Xu}, \mathrm{J}$., and H. v. Storch, 1990: Predicting the state of Southern Oscillation using principal oscillation pattern analysis. J. Climate, 3, 1316-1329.

Yasunari, T., 1989: A possible link of the QBOs between the stratosphere, troposphere and sea surface temperature in the tropics. J. Meteor. Soc. Japan., 67, 483-493. 